\title{
Overexpression of AIOLOS inhibits cell proliferation and suppresses apoptosis in Nalm-6 cells
}

\author{
YONG ZHUANG ${ }^{1}$, DONG $\mathrm{LI}^{2}$, JINQIU FU ${ }^{1}$, QING SHI ${ }^{2}$, YUANYUAN LU ${ }^{1}$ and XIULI JU \\ ${ }^{1}$ Department of Pediatrics and ${ }^{2}$ Cryomedicine Laboratory, Qilu Hospital, \\ Shandong University, Ji'nan, Shandong 250012, P.R. China
}

Received October 26, 2013; Accepted December 10, 2013

DOI: 10.3892/or.2013.2964

\begin{abstract}
The AIOLOS gene is important in the control of mature B-lymphocyte differentiation and proliferation. Previous research has shown that deregulated AIOLOS expression is associated with adult B-cell acute lymphoblastic leukemia (ALL) and chronic lymphocytic leukemia in human patients. However, the function of AIOLOS in childhood $\mathrm{B}$-cell precursor (BCP)-ALL is not fully understood. In the present study, Nalm- 6 cells were divided into three groups: the untransfected control (UT), the lentiviral vector control (LentiMock) and the AIOLOS-overexpressing (Lenti-AIOLOS) group. Lenti-AIOLOS Nalm-6 cells were constructed by lentiviral transduction, followed by cell proliferation assay, cell-cycle analysis and apoptosis assay, to evaluate the effects of AIOLOS on proliferation, cell cycle distribution and apoptosis of Nalm- 6 cells in vitro. Moreover, the expression levels of genes associated with apoptosis and the cell cycle, as well as the transcription factors IKZF1 and NF- $\kappa \mathrm{B}$, were investigated by quantitative reverse transcription-polymerase chain reaction and western blot analysis. The results showed that the proliferation of Nalm- 6 cells in the Lenti-AIOLOS group was reduced by $16 \%$ on day 8 compared with cells in the UT group $(\mathrm{P}>0.05)$. The reduction peaked at $29 \%$ on day 10 $(\mathrm{P}<0.05)$. The percentage of Nalm-6 cells in the G0/G1 phase increased from 70.4 (UT) to $84.1 \%$ (Lenti-AIOLOS) $(\mathrm{P}<0.01$ ), and the S-phase cells decreased from 20.3 (UT) to $11.7 \%$ (Lenti-AIOLOS) $(\mathrm{P}<0.01)$. Total apoptotic cells significantly decreased in AIOLOS-transfected Nalm-6 cells (10.75\%) compared with those in the Lenti-Mock (17.00\%) or UT group $(19.05 \%)(\mathrm{P}<0.01)$. In particular, the difference between the groups in the percentage of late apoptotic cells was significant (2.85 vs. $7.95 \% ; \mathrm{P}<0.01)$. In addition, overexpression of AIOLOS resulted in upregulation of $B C L-2$ and downregulation of $C C N D 3, B A X, I K Z F 1$ and $N F-\kappa B$. No changes were
\end{abstract}

Correspondence to: Professor Xiuli Ju, Department of Pediatrics, Qilu Hospital, Shandong University, 107 Wen Hua Xi Road, Ji'nan, Shandong 250012, P.R. China

E-mail: shellysdcn07@gmail.com

Key words: AIOLOS, acute leukemia, apoptosis, cell cycle, lentivirus detected on $C-M Y C$ and $P 27$. Our findings indicate that lentivirus-mediated overexpression of AIOLOS in Nalm-6 cells could inhibit cell proliferation, suppress cell apoptosis and arrest the cell cycle at the G0/G1 phase in vitro.

\section{Introduction}

Acute lymphoblastic leukemia (ALL) is the most common childhood malignancy, accounting for $\sim 25 \%$ of cancers in children $<15$ years of age; the peak onset occurs at 2-5 years of age $(1,2)$. The incidence of childhood ALL has increased yearly since the middle of the last century, and 30/1,000,000 children are diagnosed with ALL annually in China (3). Most ALLs in children arise from a B-cell precursor (BCP) $(\sim 85 \%)$, whereas the remaining $\sim 15 \%$ of cases are accounted for by T-cell acute lymphoblastic leukemias (T-ALLs) (4). The pathogenesis of ALL results from the interplay of multiple environmental and inherited factors (5). Furthermore, alterations affecting genes that encode transcriptional regulators of B-cell development and maturation are potentially important contributors to the pathogenesis of B-cell precursor acute lymphoblastic leukemia (BCP-ALL) (6).

One important group of transcription factors in hematopoietic lineages is the IKAROS family. IKAROS family genes encode a group of zinc-finger DNA-binding proteins essential in normal lymphocyte development (7-9). AIOLOS is an IKAROS family member that was first described in committed lymphoid progenitors, and is strongly upregulated as these progenitors become restricted into T- and B-lymphoid pathways (8). AIOLOS is an important regulator of B-cell differentiation, proliferation and maturation to an effector state (10). In addition, AIOLOS null mutation in mice causes B-cell hyperproliferation, elevated serum antibody levels, auto-antibody formation and development of lymphomas demonstrating tumor suppressor function (10). Deregulated AIOLOS expression has been associated with adult B-cell ALL and chronic lymphocytic leukemia (CLL) in human patients (11-13) and aberrant AIOLOS expression levels have been reported in lymphoma (14). However, the function of AIOLOS in childhood BCP-ALL is not fully understood.

BCP-ALL is a malignancy characterized by progressive accumulation of immature clonal B-cell precursors in the bone marrow (BM). Based on the function of AIOLOS in B-cell development, we sought to promote differentiation and matura- 
tion of BCP-ALL cells by delivering AIOLOS into leukemia cells. To this end, we employed a lentiviral system to stably overexpress the AIOLOS gene in Nalm-6 cells, a BCP-ALL cell line. Subsequently, we examined the effects of AIOLOS overexpression on proliferation, apoptosis and cell cycle distribution of Nalm- 6 cells in vitro. The results demonstrated that lentivirus-mediated overexpression of AIOLOS suppresses cell apoptosis and arrests the cell cycle at the G0/G1 phase, which possibly contributes to growth inhibition of Nalm-6 cells to some extent. Furthermore, these changes are possibly associated with downregulation of BAX and cyclin D3 (CCND3).

\section{Materials and methods}

Cell lines. Five leukemia cell lines were used in the present study. Two BCP-ALL cell lines (Ball-1 and Nalm-6), two T-cell leukemia cell lines (Jurkat and Molt-4), and chronic myeloblastic leukemia cell line K-562 were purchased from the American Type Culture Collection (ATCC; Manassas, VA, USA). These cell lines were cultured in standard culture medium [RPMI-1640 (Gibco, Grand Island, NY, USA) containing $10 \%$ fetal bovine serum (FBS; Gibco) and $1 \%$ penicillin-streptomycin (Gibco)] at $37^{\circ} \mathrm{C}$ in $5 \% \mathrm{CO}_{2}$ in air.

Lentiviral vector construction, virus production and transfection. For construction and identification of the AIOLOS expression plasmid, an AIOLOS insert was isolated by polymerase chain reaction (PCR) amplification from a complementary DNA (cDNA) library (Shanghai GeneChem, Shanghai, China) with two pairs of restriction primers. PCR products were sequenced (ABI Prism 3100 DNA Sequencer; Applied Biosystems, Foster City, CA, USA) and confirmed to contain the entire AIOLOS coding sequence. The insert was then cloned into the pWPT-PURO-GFP plasmid (Telebio Biomedical Co., Ltd., Shanghai, China), which was co-transfected with packaging plasmids (Telebio Biomedical Co., Ltd.) into HEK293T cells. Viral supernatant was harvested, filtered and concentrated by centrifugation. Lenti-Mock was constructed similarly, but without the AIOLOS insert. The viral concentrate was diluted in polybrene $(5 \mu \mathrm{g} / \mathrm{ml}$; Sigma, St. Louis, MO, USA) to infect Nalm- 6 cells at multiplicities of infection (MOI) of 100. A successful transduction was confirmed by visualizing enhanced green fluorescent protein (EGFP; included in the pWPT-PURO-GFP vector) after 4 days. Cells were maintained and allowed to grow for another 3-5 days, and then AIOLOS expression level was confirmed by quantitative reverse transcription-polymerase chain reaction (qRT-PCR) and western blot analysis. Virus-infected cells were selected with $8 \mu \mathrm{g} / \mathrm{ml}$ puromycin (Invitrogen, Carlsbad, CA, USA). The antibiotic-resistant clones were pooled and used for subsequent assays (Fig. 1).

Nalm- 6 cells were divided into three groups: untransfected control (UT), Lenti-Mock and AIOLOS-transfected (LentiAIOLOS) group.

Isolation of RNA and $q R T-P C R$ analysis. Total RNA was purified from each cell line or each group of Nalm-6 cells using TRIzol (Invitrogen) as per the manufacturer's protocol. We ensured that equal amounts of RNA from each subject were used. Expression levels of AIOLOS and other related

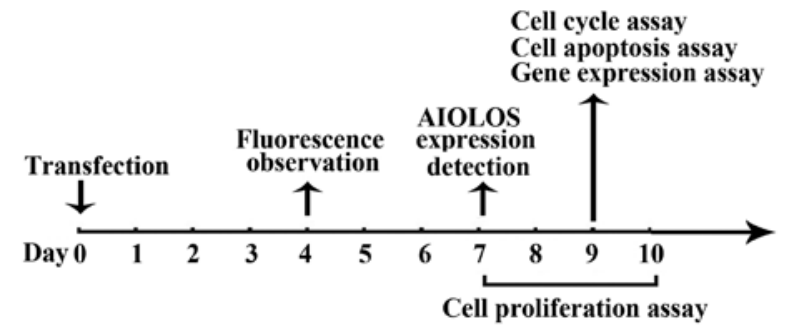

Figure 1. The main timeline of the experiment. Nalm- 6 cells were infected with pWPT-PURO-GFP-AIOLOS at multiplicities of infection (MOI) of 100 on day 0 , and the infection efficiency was detected under a fluorescence microscope after 4 days. The AIOLOS mRNA and protein expression levels in Nalm- 6 cells of the three groups were determined by qRT-PCR and western blot assays on day 7 after infection, respectively. Then, the cell numbers were counted from days 7 to 10 every day. Cell apoptosis assay, cell cycle analysis and gene expression assay were performed on day 9 .

genes were analyzed by qRT-PCR. Total RNA was reverse transcribed to cDNA with the Omniscript cDNA synthesis kit (Qiagen, Hamburg, Germany) according to the manufacturer's instructions. Approximately $0.2 \mu \mathrm{g}$ of total RNA was reverse transcribed in a $20-\mu 1$ reaction mixture containing the following components: 1X RT buffer, deoxynucleotide triphosphate $\operatorname{mix}(5 \mathrm{mM}$ each), RNase inhibitor $(10 \mathrm{U} / \mu \mathrm{l}$ RNaseOut; Invitrogen), and 4 units Omniscript RT. Samples were incubated at $37^{\circ} \mathrm{C}$ for $60 \mathrm{~min}$. The resulting cDNA was stored at $-80^{\circ} \mathrm{C}$ prior to qRT-PCR.

All reagents and primers were obtained from Bioasi Co., Ltd., Shanghai, China. $\beta$-actin was validated as an internal control.Each gene expression relative to $\beta$-actin was determined using the $2^{-\Delta \mathrm{CT}}$ method, where $\Delta \mathrm{CT}=\left(\mathrm{CT}_{\text {target gene }}-\mathrm{CT}_{\beta \text {-actin }}\right)$. qRT-PCR conditions were: $95^{\circ} \mathrm{C}$ for $4 \mathrm{~min}, 94^{\circ} \mathrm{C}$ for $15 \mathrm{sec}$, and $60^{\circ} \mathrm{C}$ for $1 \mathrm{~min}$, for a total of 40 cycles. qRT-PCR was performed using an ABI 7500 PCR system (Applied Biosystems, Foster City, CA, USA) and SYBR-Green I dye (Toyobo, Osaka, Japan). Successful amplification was defined by the presence of a single dissociation peak on the thermal melting curve. Data were analyzed with the Sequence detection software 1.4 (Applied Biosystems). Results are expressed as the normalized fold expression for each gene. Reported data are representative of at least three independent experiments. The primers used are listed in Table I.

Protein extraction and western blot analysis. Leukemia cells were harvested and the expression levels of total AIOLOS, P27, BCL-2, CCND3 and nuclear NF- $\mathrm{B}$ were analyzed. Equal amounts of proteins were separated by sodium dodecyl sulfate-polyacrylamide gel electrophoresis and then transferred to polyvinylidene difluoride membranes. Membranes were incubated with antibodies against AIOLOS, P27, BCL-2, CCND3, NF-kB or GAPDH (Abcam Inc., Cambridge, MA, USA) at $4^{\circ} \mathrm{C}$ overnight. Antibody binding was assessed by incubation with horseradish peroxidase-conjugated secondary antibodies (Beyotime Institute of Biotechnology, Shanghai, China). Chemiluminescence was detected using an ECL Plus immunoblotting detection system (Beyotime Institute of Biotechnology).

Cell proliferation assay. For cell proliferation assay, Nalm-6 cells of the UT, Lenti-Mock and Lenti-AIOLOS groups were 
Table I. Primer sequences (5'-3') used for qRT-PCR.

\begin{tabular}{|c|c|c|}
\hline Gene & Primer sequences & $\begin{array}{c}\text { Product } \\
\text { length } \\
\text { (bp) }\end{array}$ \\
\hline$\beta$-actin & $\begin{array}{l}\text { F: 5'-GGACATCCGCAAAGACCTGTA-3' } \\
\text { R: 5'-GCATCCTGTCGGCAATGC-3' }\end{array}$ & 80 \\
\hline AIOLOS & $\begin{array}{l}\text { F: 5'-GCCCTTCAAGTGTTTCACCAA-3' } \\
\text { R: 5'-GCCTTTCCAGCCAGACAAATAT-3' }\end{array}$ & 90 \\
\hline$B C L-2$ & $\begin{array}{l}\text { F: 5'-GCTGGGAGAACAGGGTACGA-3' } \\
\text { R: 5'-CCTCTGCGACAGCTTATAATGGA-3' }\end{array}$ & 80 \\
\hline$B A X$ & $\begin{array}{l}\text { F: 5'-CTTGTTGCCCAGGCTTGAGT-3' } \\
\text { R: 5'-GCAGGAGAATCGCTTGAACCT-3' }\end{array}$ & 81 \\
\hline CCND3 & $\begin{array}{l}\text { F: 5'-GAGGTGCAATCCTCTCCTCG-3' } \\
\text { R: 5'-TCACATACCTCCTCGTCAGGT-3' }\end{array}$ & 87 \\
\hline$C-M Y C$ & $\begin{array}{l}\text { F: 5'-TCTCCGTCCTCGGATTCTCT-3' } \\
\text { R: 5'-TTCCTCCTCAGAGTCGCTGC-3' }\end{array}$ & 85 \\
\hline$P 27$ & $\begin{array}{l}\text { F: 5'-TCCGGCTAACTCTGAGGACA-3' } \\
\text { R: 5'-GAAGAATCGTCGGTTGCAGG-3' }\end{array}$ & 81 \\
\hline$I K Z F 1$ & $\begin{array}{l}\text { F: 5'-AGAAGCCACACTGGAGAACG-3' } \\
\text { R: 5'-GCAGAGGTGGCATTTGAAGG-3' }\end{array}$ & 83 \\
\hline$N F-\kappa B$ & $\begin{array}{l}\text { F: 5'-TCCATATTTGGGAAGGCCTGA-3' } \\
\text { R: 5'-GGTATGGGCCATCTGCTGT-3' }\end{array}$ & 89 \\
\hline
\end{tabular}

F, forward; R, reverse.

plated onto a 6-well culture plate at a density of $5 \times 10^{5}$ cells/well on day 7 after transfection. Viable cells were counted on days 8,9 and 10, respectively. Each time-point was counted in triplicate. Results are given as the fold-change relative to the initial cell numbers $\left(5 \times 10^{5}\right)$, which were set at fold-change $=1$. Growth curves were plotted with the means of each experiment, and error bars represent the standard errors of means (SEM).

Cell cycle analysis. A total of $1 \times 10^{6}$ cells from each group were washed three times and resuspended in $\sim 50 \mu \mathrm{l}$ PBS. Resuspended cells were added into the tube containing $1 \mathrm{ml}$ of ice cold $70 \%$ ethanol dropwise while vortexing at medium speed. The tubes were frozen at $-20^{\circ} \mathrm{C}$ for $3 \mathrm{~h}$ prior to staining. Subsequently, cells were washed and treated with $200 \mu \mathrm{l}$ of Muse $^{\mathrm{TM}}$ Cell Cycle reagent (Millipore Corp., Bedford, MA, USA) according to the manufacturer's protocol. After $30 \mathrm{~min}$ of incubation at room temperature in the dark, cell suspension samples were transferred into $1.5 \mathrm{ml}$ microcentrifuge tubes and analyzed using the Muse ${ }^{\mathrm{TM}}$ Cell Analyzer. Results are expressed as percentage of cells in each cell cycle phase and error bars represent SEM.

Cell apoptosis assay. Nalm-6 cell apoptosis was assayed using the Muse ${ }^{\mathrm{TM}}$ Annexin V and Dead Cell kit (Millipore) according to the user's guide. A total of $1 \times 10^{5}$ cells from each group were collected by centrifugation (2,000 rpm, $5 \mathrm{~min})$ and washed with PBS. Cells were resuspended in PBS with $1 \%$ bovine serum albumin and $1 \%$ FBS, mixed with the Muse ${ }^{\mathrm{TM}}$ Annexin V
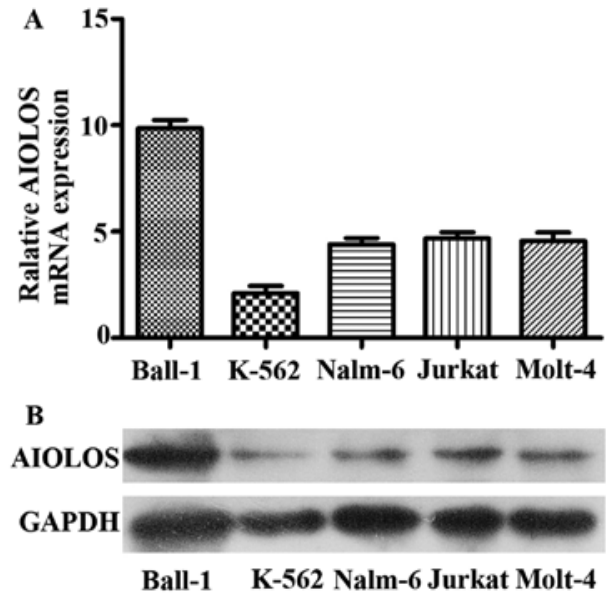

Figure 2. Expression levels of AIOLOS in leukemia cell lines. (A) qRT-PCR showed the relative transcript levels of AIOLOS in five leukemia cell lines, Ball-1, K-562, Nalm-6, Jurkat and Molt-4. $\beta$-actin was used as internal control. (B) Western blot results showed that AIOLOS was expressed in the five leukemia cell lines. GAPDH served as loading control. Error bars indicate SEM ( $n=3$ experiments).

and Dead Cell reagent, and then incubated for $20 \mathrm{~min}$ at room temperature in the dark. Assay results were measured using the Muse ${ }^{\mathrm{TM}}$ Cell Analyzer. Results are expressed as percentage of apoptotic cells and error bars represent SEM.

Statistical analysis. All experiments were performed three times and the differences between the experimental and control cells were analyzed by the Student's t-test. The data are presented as means \pm SEM of three independent experiments and $\mathrm{P}<0.05$ was considered to indicate a statistically significant result.

\section{Results}

AIOLOS is differentially expressed in five leukemia cell lines. AIOLOS expression level was quantified by qRT-PCR and western blot analysis in five human leukemia cell lines. As indicated in Fig. 2A, although expression levels varied, AIOLOS mRNA was expressed in each cell line. Among the cell lines, Ball-1 expressed the highest level of AIOLOS, whereas K-562 expressed the lowest. Nalm-6, Jurkat and Molt-4 exhibited moderate expression levels of AIOLOS. Furthermore, we performed western blot analysis to verify the level of AIOLOS protein in the five cell lines. The results were consistent with those of qRT-PCR (Fig. 2B). BCP-ALL is the main form of ALL in children, therefore Nalm- 6 cells, a BCP-ALL cell line, were chosen for a series of functional experiments.

Identification of recombinant plasmid pWPT-PURO-GFPAIOLOS and overexpression of AIOLOS by stable transfection in Nalm- 6 cells. To upregulate AIOLOS expression in Nalm-6 cells, the entire AIOLOS coding sequence was cloned into a lentivirus vector pWPT-PURO-GFP. The resulting construct was verified by DNA sequencing. The detected sequence was identical to the known AIOLOS sequence in GenBank (NM_012481.4). Western blot analysis revealed a 58-kDa band in the cell extracts, which was the expected size of an AIOLOS protein (Fig. 3H). 

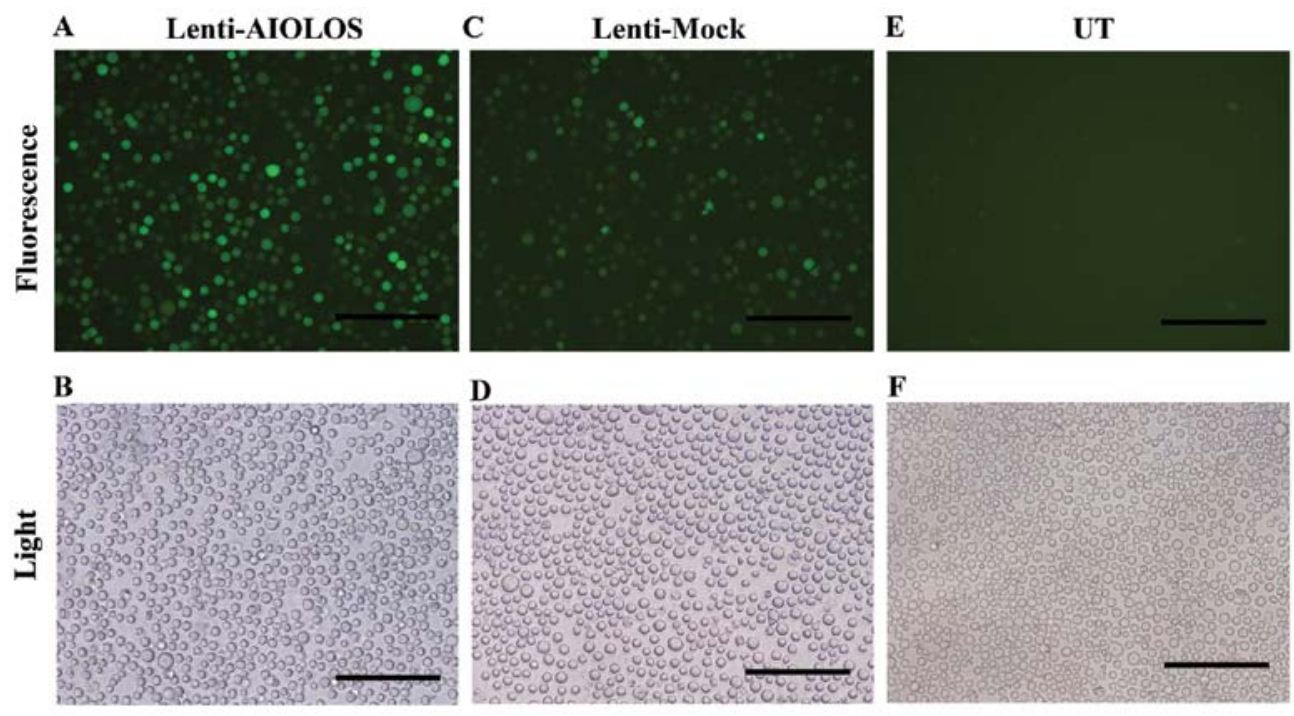

F

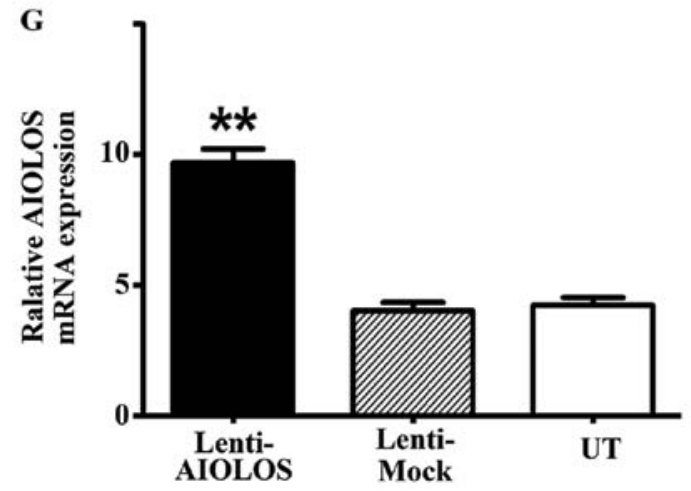

H

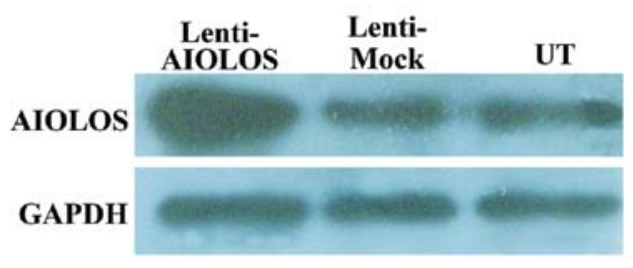

Figure 3. Determination of lentiviral transduction efficiency and expression level of AIOLOS in Nalm-6 cells of the Lenti-AIOLOS group. (A-F) Lentiviral transduction efficiency in Nalm- 6 cells. Transduction efficiency was estimated 4 days after infection at MOI=100. GFP expression was observed under fluorescence microscopy (A, C and E) or light microscopy (B, D and F). (A and B) AIOLOS-transfected (Lenti-AIOLOS) group. (C and D) Lentiviral vector control (Lenti-Mock). (E and F) Untransfected control (UT). Scale bar, $200 \mu \mathrm{m}$. (G) qRT-PCR showed relative transcript levels of AIOLOS in the Lenti-AIOLOS, the Lenti-Mock and the UT group. $\beta$-actin was used as internal control. $(\mathrm{H})$ Western blot results showed that AIOLOS was expressed in Nalm- 6 cells of the three groups. GAPDH served as loading control. Error bars indicate SEM ( $\mathrm{n}=3$ experiments). ${ }^{* *} \mathrm{P}<0.01$ Lenti-AIOLOS vs. Lenti-Mock.

Nalm-6 cells were infected with lentivirus vector pWPTPURO-GFP-AIOLOS. As control, Nalm-6 cells were either infected with a lentivirus vector expressing GFP or not. At precisely $96 \mathrm{~h}$ after infection of Nalm-6 cells, the infection efficiency was detected using a fluorescence microscope. A large number of cells emitted bright green fluorescence, which represented high infection efficiency (Fig. 3A-F). Cells were maintained and allowed to grow for 3-5 days. AIOLOS mRNA and protein expression levels in Nalm- 6 cells of the three groups were determined by qRT-PCR and western blot assays on day 7 after infection. qRT-PCR demonstrated that the expression level of AIOLOS mRNA in Nalm- 6 cells of the Lenti-AIOLOS group markedly increased compared with that of the Lenti-Mock group and UT group, which was consistent with the increase in AIOLOS protein expression (Fig. 3G and $\mathrm{H}$ ). No significant difference between cells of the LentiMock group and the UT group was observed. These results indicated that the stable transfection of pWPT-PURO-GFPAIOLOS upregulated AIOLOS expression in Nalm- 6 cells.

Overexpression of AIOLOS suppresses the proliferation of Nalm-6 cells in vitro. To assess the effect of AIOLOS overexpression on cell proliferation of Nalm- 6 cells, cell numbers

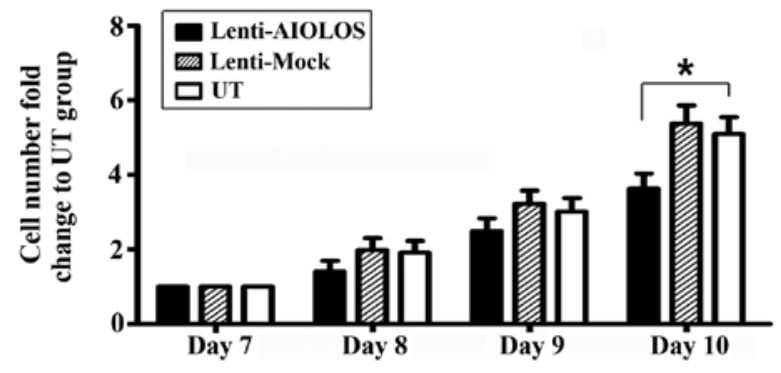

Figure 4. Effect of AIOLOS on the proliferation of Nalm-6 cells. Cell numbers of Nalm- 6 cells of the three groups were counted on days 7, 8, 9 and 10. Each time-point was counted in triplicate. Results are given as the fold-change relative to the initial cell number $\left(5 \times 10^{5}\right)$, which was set at foldchange $=1$. Proliferation of Nalm- 6 cells in the Lenti-AIOLOS group was reduced by $16 \%$ on day 8 compared with that in UT group cells $(\mathrm{P}>0.05)$. The reduction peaked at $29 \%$ on day $10\left({ }^{*} \mathrm{P}<0.05\right)$. The Lenti-Mock group had the same growth ability as the UT group $(\mathrm{P}>0.05)$. Error bars represent the standard errors of means (SEM) ( $\mathrm{n}=3$ experiments).

were counted on days 8,9 and 10 after transfection. As shown in Fig. 4, proliferation of Nalm-6 cells in the Lenti-AIOLOS group was reduced by $16 \%$ on day 8 compared with that in the UT group cells $(P>0.05)$. The reduction peaked at $29 \%$ 
A

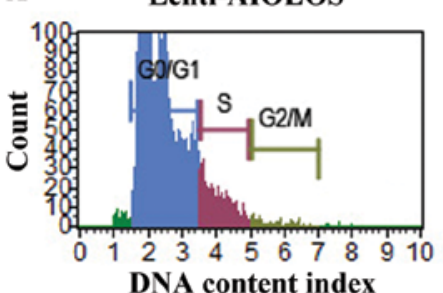

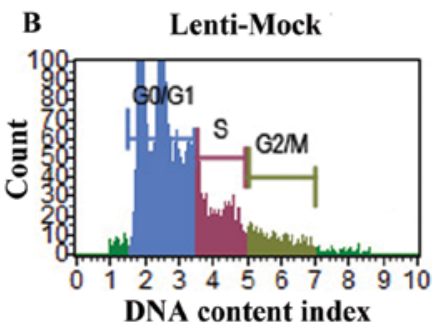

C

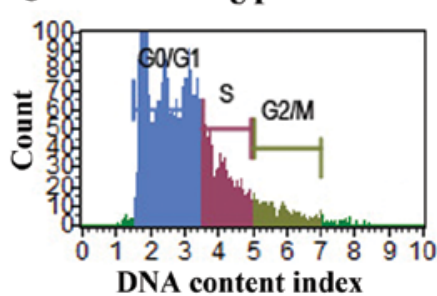

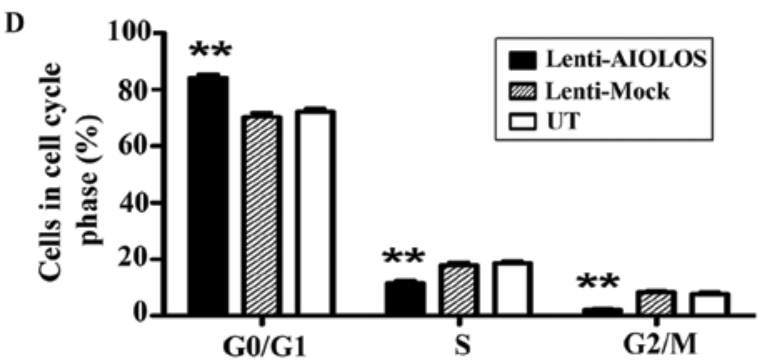

Figure 5. Effect of AIOLOS on cell cycle distribution in Nalm-6 cells. (A-C) Flow cytometric analysis demonstrated that the fraction of G0/G1 phase cells increased and the proportion of S-phase and G2/M phase cells decreased in the Nalm-6 cells with increased expression of AIOLOS. (D) The proportions of G0/G1 increased and G2/M and S phase cells decreased in Nalm- 6 cells of the Lenti-AIOLOS group compared with those in the Lenti-Mock group ( ${ }^{* *} \mathrm{P}<0.01$, Lenti-AIOLOS vs. Lenti-Mock). Error bars represent the standard errors of means (SEM) ( $\mathrm{n}=3$ experiments).
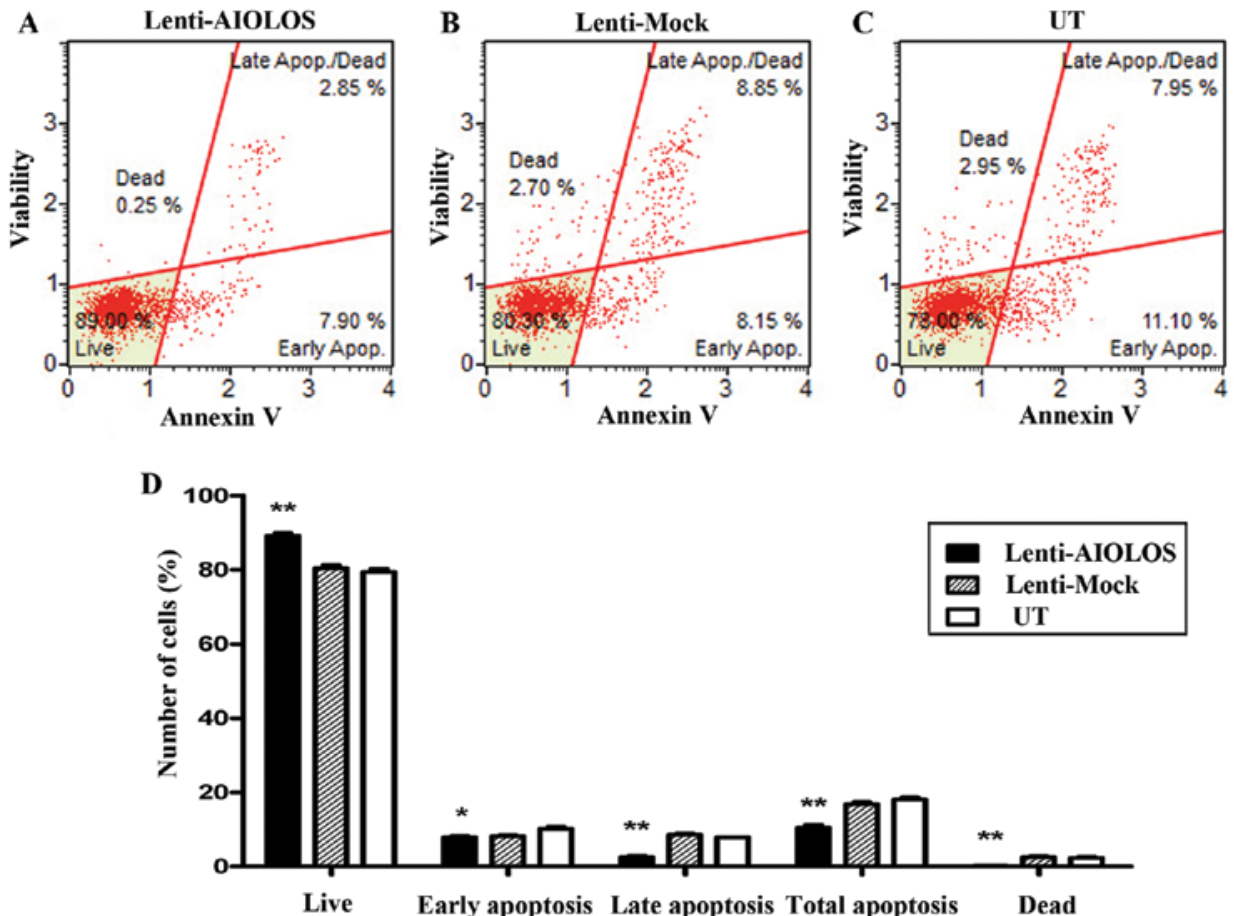

Figure 6. Effect of AIOLOS on Nalm-6 cell apoptosis. (A-C) Flow cytometric analysis demonstrated that the proportion of total apoptotic cells decreased in the Nalm-6 cells with increased expression of AIOLOS. (D) Total apoptotic cells significantly decreased in AIOLOS-transfected Nalm-6 cells compared with those in the Lenti-Mock or UT group $\left.{ }^{* *} \mathrm{P}<0.01\right)$. Specifically, the difference between AIOLOS-transfected Nalm- 6 cells and UT Nalm- 6 cells in the percentage of early apoptotic cells was slight ("P<0.05), whereas the difference between them in the percentage of late apoptotic cells was significant $\left.{ }^{(* *} \mathrm{P}<0.01\right)$. Error bars represent the standard errors of means (SEM) ( $\mathrm{n}=3$ experiments).

on day $10(\mathrm{P}<0.05)$. The Lenti-Mock group exhibited similar growth ability as the UT group ( $\mathrm{P}>0.05)$. The results indicated that overexpression of AIOLOS suppressed the growth of Nalm-6 cells to some degree.

Upregulation of AIOLOS arrests Nalm- 6 cells at the GO/GI phase. The different proliferation rates of Nalm- 6 cells infected with Lenti-AIOLOS vs. control Nalm-6 cells may partly be due to the differences in cell cycle regulation. Therefore, the cell cycle of Nalm-6 cells in the Lenti-AIOLOS, Lenti-Mock and UT groups were characterized by fluorescence-activated cell sorting (FACS) analysis on day 9 after transfection. As shown in Fig. 5, no significant difference between the LentiMock and Nalm-6 cells was observed $(\mathrm{P}>0.05)$. However, 


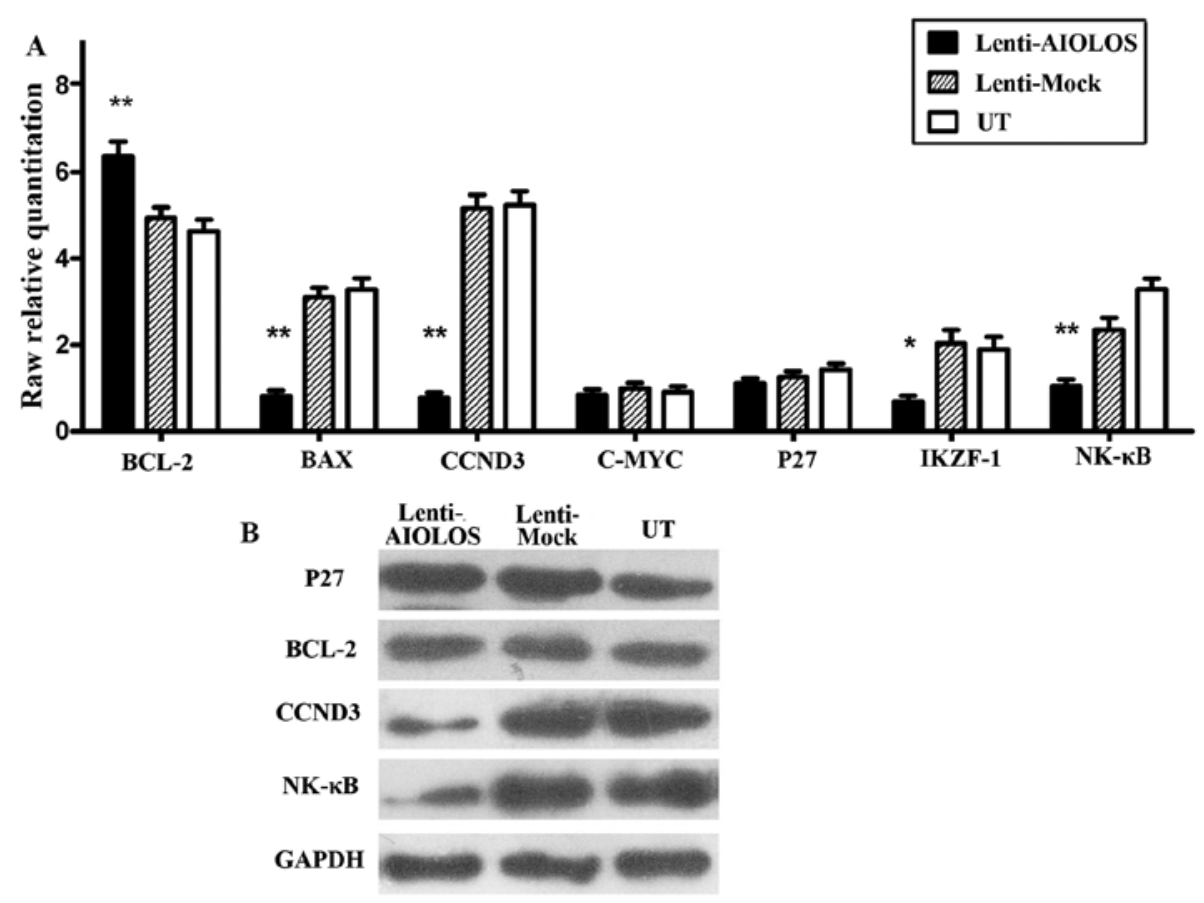

Figure 7. Effect of AIOLOS on the expression levels of apoptosis and cell cycle-related genes in Nalm- 6 cells. (A) qRT-PCR shows relative transcript levels of $B C L-2, B A X, C C N D 3, C-M Y C, P 27, I K Z F 1$ and $N F-\kappa B$ in Nalm-6 cells of the three groups. $B A X\left({ }^{* *} \mathrm{P}<0.01\right)$ and $C C N D 3\left({ }^{* *} \mathrm{P}<0.01\right)$ were markedly reduced in Nalm- 6 cells of the Lenti-AIOLOS group compared with those in the control groups, while the expression level of $B C L-2(" \mathrm{P}<0.05)$ was increased. Minimal change was observed in the expression of $C$-MYC $(\mathrm{P}>0.05)$ and $P 27(\mathrm{P}>0.05)$. Downregulation of $I K Z F 1\left({ }^{*} \mathrm{P}<0.05\right)$ and $N F-\kappa B\left({ }^{* *} \mathrm{P}<0.01\right)$ was confirmed in the Lenti-AIOLOS group compared with the control groups. $\beta$-actin was used as internal control. Error bars represent the standard errors of means (SEM) ( $n=3$ experiments). (B) Western blot results showed the expression levels of P27, BCL-2, CCND3 and NF- $\mathrm{BB}$ in Nalm- 6 cells of the three groups. Decreased expression of CCND3 and NF-KB and minimal change in BCL-2 and P27 were observed. GAPDH served as loading control.

the percentage of Nalm-6 cells in G0/G1 phase increased from 70.4 (UT) to $84.1 \%$ (Lenti-AIOLOS) $(\mathrm{P}<0.01)$, and the S-phase cells decreased from 20.3 (UT) to $11.7 \%$ (LentiAIOLOS) $(\mathrm{P}<0.01)$. The difference between Nalm- 6 cells of the Lenti-AIOLOS group and the UT group in G2/M phase was significant $(2.2$ vs. $7.3 \%$; $\mathrm{P}<0.01)$. The data indicated that upregulation of AIOLOS expression arrested Nalm- 6 cells at the G0/G1 phase, which possibly contributed to the growth inhibition of Nalm- 6 cells.

Overexpression of AIOLOS suppresses cell apoptosis of Nalm-6 cells. To determine whether AIOLOS overexpression results in apoptosis in Nalm-6 cells, we used a Muse ${ }^{\mathrm{TM}}$ Annexin V and Dead Cell kit to measure the changes in cell apoptosis on day 9. As shown in Fig. 6, total apoptotic cells were significantly decreased in AIOLOS-transfected Nalm-6 cells $(10.75 \%)$ compared with those in the Lenti-Mock (17.00\%) or UT groups $(19.05 \%)(\mathrm{P}<0.01)$. In particular, the difference between AIOLOS-transfected Nalm-6 cells and UT Nalm-6 cells in the percentage of early apoptotic cells was minimal ( 7.90 vs. $11.10 \% ; \mathrm{P}<0.05)$, whereas the difference between the cell groups in the percentage of late apoptotic cells was significant $(2.85$ vs. $7.95 \%$; $\mathrm{P}<0.01)$. These data suggest that overexpression of AIOLOS suppresses cell apoptosis in Nalm-6 cells.

Effect of AIOLOS on the expression levels of apoptosis and cell cycle-related genes in Nalm- 6 cells. To further explore the underlying mechanism in the above-mentioned changes of biological behaviors, we examined the expression of genes associated with apoptosis and cell cycle in response to AIOLOS overexpression by qRT-PCR (Fig. 7A) and western blot analysis (Fig. 7B). We also examined the expression level of $I K Z F I$, another IKAROS family member. qRT-PCR showed that the levels of pro-apoptotic gene $B A X(\mathrm{P}<0.01)$, and cell-cycle-associated gene CCND3 $(\mathrm{P}<0.01)$, were markedly reduced in Nalm- 6 cells of the Lenti-AIOLOS group compared with that in the control groups, while the expression level of anti-apoptotic gene $B C L-2(\mathrm{P}<0.05)$ was increased. However, minimal change was observed in the expression of $C-M Y C(\mathrm{P}>0.05)$ and $P 27(\mathrm{P}>0.05)$. In addition, downregulation of IKZFI $(\mathrm{P}<0.05)$ and $N F-\kappa B(\mathrm{P}<0.01)$ was confirmed in the Lenti-AIOLOS group compared with the control groups. Consistent with qRT-PCR, decreased expressions of CCND3 and NF- $\kappa \mathrm{B}$, as well as steady expression level of P27, were observed by western blot analysis. No significant change in the expression of BCL-2 protein was observed, which was inconsistent with qRT-PCR results.

\section{Discussion}

The AIOLOS transcription factor IKZF3, encoded by the gene IKZF3 located on chromosome 17q12, is a member of the IKAROS family of zinc-finger proteins, and is important in the control of mature B-lymphocyte differentiation and proliferation. Thus, to better understand the function of AIOLOS in the pathogenesis of BCP-ALL, we chose the BCP-ALL cell line Nalm- 6 for a series of functional studies. 
At least 16 different isoforms of AIOLOS resulting from alternate splicing have different cellular localizations and the ability to change the localization of other IKAROS members (15). Moreover, the AIO1 transcript represents more than $80 \%$ of the AIOLOS isoforms in B-cells (16). To mimic the isoforms and cellular localizations of AIOLOS in B-cells, we constructed a plasmid pWPT-PURO-GFP-AIOLOS containing the entire AIOLOS coding sequence and performed lentivirus-mediated transduction in Nalm- 6 cells to create a stable transfection cell line. Our results indicated that Nalm- 6 cells were successfully transduced with lentivirus and AIOLOS was successfully overexpressed in Nalm- 6 cells.

Previous studies have shown that loss of AIOLOS in mice results in increased pre-B and immature B cells precursors (10). In the present study, we showed that overexpression of AIOLOS could suppress the proliferation of Nalm-6 in vitro. To explore the potential mechanisms underlying the action of AIOLOS in Nalm-6 cell growth, cell cycling was characterized by FACS analysis. Data indicated that upregulation of AIOLOS expression arrested Lenti-AIOLOS cell cycling at the G0/G1 phase. This result supports the hypothesis that AIOLOS inhibits Nalm- 6 cell proliferation through cell cycle regulation. Proliferation of pre-B cells requires both Ccnd3 and c-Myc $(17,18)$. Recent studies have demonstrated that cell cycle arrest in pre-B cells is associated with downregulation of c-Myc and Cend 3 and induction of p27 in mice (19). We then investigated whether overexpression of AIOLOS in Nalm-6 cells could affect these cell cycle-associated genes. However, we only found downregulation of CCND3, whereas C-MYC and P27 expressions showed minimal change. We attributed this conflict to two possible reasons; first, AIOLOS directly represses C-MYC, which in turn leads to upregulation of P27 and downregulation of CCND3 (19). This process follows a time sequence. Therefore, C-MYC and P27 returned to their original expression levels in our experiment. Second, unlike in mice, AIOLOS could possibly arrest cell cycle in pre-B cells via other pathways in humans.

AIOLOS reportedly controls $\mathrm{T}$ cell death by regulating the expression and localization of the anti-apoptotic molecule Bcl-2 (20), suggesting the possibility that evasion of apoptotic cell death is a common mechanism through which IKAROS family proteins participate in leukemogenesis. In the present study, we confirmed that overexpression of AIOLOS in Nalm- 6 cell inhibited cell apoptosis, consistent with previous research demonstrating that disruption of AIOLOS causes B cells to be more prone to apoptosis (21). Previous studies have found that the lack of AIOLOS accelerates premature B cell apoptosis mediated by BCR signaling through elevation in cytochrome $c$ release (22). In the present study, we investigated whether the disruption of apoptosis-related genes BCL-2 and BAX contributed to apoptosis inhibition of Nalm- 6 cells by AIOLOS overexpression. Although BCL-2 protein showed minimal change in western blot analysis, BCL-2 mRNA was significantly upregulated by qRT-PCR. This inconsistency is possibly caused by complicated posttranscriptional regulations. These results indicated that increased level of BCL-2 and decreased level of BAX is a possible reason for the decreased apoptosis in Nalm- 6 cells. However, further research is required to explore the complete mechanism.
IKAROS is a master regulator during the early stages of lymphocyte ontogeny and differentiation $(9,23)$. NF- $\kappa B$ is widely recognized as a key positive regulator of cancer cell proliferation and survival via its ability to transcriptionally activate many pro-survival and anti-apoptotic genes, such as XIAP, Bcl-2, Bcl-Xl, IкB $\alpha$, cIAP-1, cIAP-2 and survivin (24). Previous studies showing that IKAROS and NF- $\mathrm{KB}$ have the potential to stimulate AIOLOS expression suggest that these transcription factors are possible upstream AIOLOS effectors (25). Thus, the expression levels of IKAROS and NF- $\kappa B$ mRNA were detected by qRT-PCR, and NF- $\mathrm{kB}$ protein was detected by western blot analysis. Notably, both IKAROS and NF- $\mathrm{KB}$ expression decreased following AIOLOS overexpression. We speculate that since IKAROS and NF- $\kappa$ B could stimulate AIOLOS expression, highly expressed levels of AIOLOS could exhibit feedback inhibition to IKAROS and NF- $\mathrm{KB}$ for maintenance of homeostasis.

In summary, the present study explored the function of the transcription factor AIOLOS in biological behaviors in human BCP-ALL cell line for the first time, which will provide foundation for further research on the pathogenesis of BCP-ALL. Our results indicated that upregulation of AIOLOS expression in Nalm- 6 cells inhibited cell proliferation, suppressed cell apoptosis and arrested cell cycle at the G0/G1 phase. The present study emphasized the hypothesis that disruption of AIOLOS may be critical for BCP-ALL pathogenesis. However, the mechanism through which AIOLOS interacts with other proliferation and apoptosis regulators is poorly understood. Characterizing these potential genetic interactions will be of future interest.

\section{Acknowledgements}

The present study was supported by grants from the Shandong Province Natural Science Foundation (ZR2011HM007 and 2013GSF11812) and the Innovation Fund Project of Shandong University (2012ZD023).

\section{References}

1. Eden T: Aetiology of childhood leukaemia. Cancer Treat Rev 36: 286-297, 2010.

2. Kaatsch P: Epidemiology of childhood cancer. Cancer Treat Rev 36: 277-285, 2010.

3. Howard SC, Metzger ML, Wilimas JA, Quintana Y, Pui CH, Robison LL and Ribeiro RC: Childhood cancer epidemiology in low-income countries. Cancer 112: 461-472, 2008.

4. Pui CH, Campana D and Evans WE: Childhood acute lymphoblastic leukaemia - current status and future perspectives. Lancet Oncol 2: 597-607, 2001.

5. Belson M, Kingsley B and Holmes A: Risk factors for acute leukemia in children: a review. Environ Health Perspect 115: 138-145, 2007.

6. Cobaleda C and Sanchez-Garcia I: B-cell acute lymphoblastic leukaemia: towards understanding its cellular origin. Bioessays 31: 600-609, 2009.

7. Kelley CM, Ikeda T, Koipally J, Avitahl N, Wu L, Georgopoulos K and Morgan BA: Helios, a novel dimerization partner of Ikaros expressed in the earliest hematopoietic progenitors. Curr Biol 8: 508-515, 1998

8. Morgan B, Sun L, Avitahl N, Andrikopoulos K, Ikeda T, Gonzales E, Wu P, Neben S and Georgopoulos K: Aiolos, a lymphoid restricted transcription factor that interacts with Ikaros to regulate lymphocyte differentiation. EMBO J 16: 2004-2013, 1997.

9. Georgopoulos K, Winandy S and Avitahl N: The role of the Ikaros gene in lymphocyte development and homeostasis. Ann Rev Immunol 15: 155-176, 1997. 
10. Wang JH, Avitahl N, Cariappa A, Friedrich C, Ikeda T, Renold A, Andrikopoulos K, Liang L, Pillai S, Morgan BA and Georgopoulos K: Aiolos regulates B cell activation and maturation to effector state. Immunity 9: 543-553, 1998.

11. Nakase K, Ishimaru F, Avitahl N, Dansako H, Matsuo K, Fujii K, Sezaki N, Nakayama H, Yano T, Fukuda S, Imajoh K, Takeuchi M, Miyata A, Hara M, Yasukawa M, Takahashi I, Taguchi H, Matsue K, Nakao S, Niho Y, Takenaka K, Shinagawa K, Ikeda K, Niiya K and Harada M: Dominant negative isoform of the Ikaros gene in patients with adult B-cell acute lymphoblastic leukemia. Cancer Res 60: 4062-4065, 2000.

12. Nuckel H, Frey UH, Sellmann L, Collins CH, Duhrsen U and Siffert W: The IKZF3 (Aiolos) transcription factor is highly upregulated and inversely correlated with clinical progression in chronic lymphocytic leukaemia. Br J Haematol 144: 268-270, 2009.

13. Billot K, Soeur J, Chereau F, Arrouss I, Merle-Beral H, Huang ME, Mazier D, Baud V and Rebollo A: Deregulation of Aiolos expression in chronic lymphocytic leukemia is associated with epigenetic modifications. Blood 117: 1917-1927, 2011.

14. Antica M, Cicin-Sain L, Kapitanovic S, Matulic M, Dzebro S and Dominis M: Aberrant Ikaros, Aiolos, and Helios expression in Hodgkin and non-Hodgkin lymphoma. Blood 111: 3296-3297, 2008.

15. Caballero R, Setien F, Lopez-Serra L, Boix-Chornet M, Fraga MF, Ropero S, Megias D, Alaminos M, Sanchez-Tapia EM, Montoya MC, Esteller M, Gonzalez-Sarmiento R and Ballestar E: Combinatorial effects of splice variants modulate function of Aiolos. J Cell Sci 120: 2619-2630, 2007.

16. Duhamel M, Arrouss I, Merle-Beral H and Rebollo A: The Aiolos transcription factor is up-regulated in chronic lymphocytic leukemia. Blood 111: 3225-3228, 2008.
17. Cooper AB, Sawai CM, Sicinska E, Powers SE, Sicinski P, Clark MR and Aifantis I: A unique function for cyclin D3 in early B cell development. Nat Immunol 7: 489-497, 2006.

18. Habib T, Park H, Tsang M, de Alboran IM, Nicks A, Wilson L, Knoepfler PS, Andrews S, Rawlings DJ, Eisenman RN and Iritani BM: Myc stimulates B lymphocyte differentiation and amplifies calcium signaling. J Cell Biol 179: 717-731, 2007.

19. Ma S, Pathak S, Mandal M, Trinh L, Clark MR and Lu R: Ikaros and Aiolos inhibit pre-B-cell proliferation by directly suppressing c-Myc expression. Mol Cell Biol 30: 4149-4158, 2010.

20. Romero F, Martinez AC, Camonis J and Rebollo A: Aiolos transcription factor controls cell death in T cells by regulating Bcl-2 expression and its cellular localization. EMBO J 18: 3419-3430, 1999.

21. Narvi E, Nera KP, Terho P, Mustonen L, Granberg J and Lassila O: Aiolos controls gene conversion and cell death in DT40 B cells. Scand J Immunol 65: 503-513, 2007.

22. Kikuchi H, Yamashita K, Nakayama M, Toyonaga K, Tsuneyoshi I, Takasaki M and Nakayama T: Lacking of Aiolos accelerates pre-mature B cell apoptosis mediated by BCR signaling through elevation in cytochrome $\mathrm{c}$ release. Biochim Biophys Acta 1793: 1304-1314, 2009.

23. Georgopoulos K, Bigby M, Wang JH, Molnar A, Wu P, Winandy S and Sharpe A: The Ikaros gene is required for the development of all lymphoid lineages. Cell 79: 143-156, 1994.

24. Sethi G, Ahn KS and Aggarwal BB: Targeting nuclear factorkappa $\mathrm{B}$ activation pathway by thymoquinone: role in suppression of antiapoptotic gene products and enhancement of apoptosis. Mol Cancer Res 6: 1059-1070, 2008.

25. Ghadiri A, Duhamel M, Fleischer A, Reimann A, Dessauge F and Rebollo A: Critical function of Ikaros in controlling Aiolos gene expression. FEBS Lett 581: 1605-1616, 2007. 